\title{
Combining Fairness and Optimality when Selecting and Allocating Projects
}

\author{
Khaled Belahcène $^{1}$, Vincent Mousseau ${ }^{2}$ and Anaëlle Wilczynski ${ }^{2}$ \\ ${ }^{1}$ Université de technologie de Compiègne, CNRS, Heudiasyc \\ ${ }^{2}$ MICS, CentraleSupélec, Université Paris-Saclay \\ khaled.belahcene@hds.utc.fr, \{vincent.mousseau, anaelle.wilczynski\}@ centralesupelec.fr
}

\begin{abstract}
We consider the problem of the conjoint selection and allocation of projects to a population of agents, e.g. students are assigned papers and shall present them to their peers. The selection can be constrained either by quotas over subcategories of projects, or by the preferences of the agents themselves. We explore fairness and optimality issues and refine the analysis of the rank-maximality and popularity optimality concepts. We show that they are compatible with reasonable fairness requirements related to rank-based envy-freeness and can be adapted to select globally good projects according to the preferences of the agents.
\end{abstract}

\section{Introduction}

When allocating indivisible resources to a group of agents, optimality and fairness are key considerations in order to satisfy the agents with their assigned resources [Bouveret et al., 2016]. However, when not all resources are to be assigned, the choice of the assigned resources may be particularly important. Many real-world situations can illustrate this issue.

A first context concerns a University course where students must be assigned to projects they have to work on, and present to their peers at the end of the course. Usually, an allocation that takes into account the preferences of the students is desirable in order to motivate them to work. An optimal allocation of projects to the students should maximize the global satisfaction of the students. This allocation must also be fair otherwise students could complain about the assignment. However, the choice of selected projects can also be fundamental for pedagogical reasons: not all students should present similar projects since it is expected that even attendants will learn from the presentations. Therefore, some diversity constraints according to the topics of the projects must be considered.

Another example concerns the organization of a conference with invited speakers. Suppose each of the speakers must be assigned a one hour time slot from Monday to Wednesday (9:00-19:00). In such a context, speakers can express preferences over time slots (e.g. preferably in the morning or early in the conference). The decisions to make involve selecting time slots for presentations, and the allocation of a time slot for each speaker. Solving such selection/allocation should account for the preferences of speakers both for the conference timetable, and for the time of their presentation.

The two preceding examples involve agents and many resources (more than the number of agents), referred to as projects, to be assigned to agents by a central authority. The assignment must be optimal and fair for the agents, and at the same time the set of assigned projects should be selected regarding balance constraints with respect to groups of projects (topics, days) or preferences of the agents. Hence it can be seen as a combination of multiwinner voting [Elkind et al., 2017; Faliszewski et al., 2017] (we want a good selection of projects) and resource allocation problems (we want a fair and optimal assignment of projects to agents).

In this paper, we focus on house allocation problems [Hylland and Zeckhauser, 1979; Abdulkadiroğlu and Sönmez, 1998], where each agent must be assigned exactly one project. Optimality questions have been widely investigated, especially with respect to Pareto-optimality [Abraham et al., 2004] and its refinements, such as rank-maximality [Irving, 2003; Irving et al., 2006] and popularity [Abraham et al., 2007; Cseh, 2017]. Concerning fairness, the most classical criterion, envy-freeness [Foley, 1967; Varian, 1974], requires that no agent prefers the project assigned to another agent to her own assigned project. This criterion is too demanding if the number of projects equals the number of agents. Hence, some relaxations of envy-freeness have been proposed [Beynier et al., 2019]. However, envy-freeness is more interesting when there are more projects than agents [Gan et al., 2019].

We focus in this article on a relaxation of envy-freeness, called rank-envy-freeness, which requires that no agent prefers the project assigned to another agent whereas she ranked this project in a better position in her preference ranking. This notion is present in the favoring higher ranks concept introduced by Ramezanian and Feizi [2020], was implicitly used by Kojima and Ünver [2014], and is equivalent to no envy by rank in random assignment [Harless, 2018]. We propose a generalization of rank-envy-freeness and introduce another concept, frustration-freeness, which relates both allocation and selection issues. Frustration-freeness imposes that no agent prefers an unassigned project to her assigned project. Focusing on the personal satisfaction of an agent, it can be viewed as a fairness concept, but also as an optimality concept because it asks for no project waste. Whereas optimality and 
fairness are often incompatible [Bouveret and Lang, 2008], we show that rank-envy-freeness and frustration-freeness are necessary to characterize rank-maximality and popularity.

For choosing an adequate set of selected projects at the same time as a good allocation of projects to agents, we investigate two ways: considering hard diversity constraints represented by lower and upper quotas over categories, or considering the preferences of the agents over the selected projects.

Diversity constraints are relevant in multiwinner voting when a committee with particular attributes is sought [Brams, 1990; Bredereck et al., 2018]. In resource allocation, it has been investigated, e.g., in public housing allocation [Benabbou et al., 2018], and in course allocation [Cechlárová and Fleiner, 2017] where courses have capacities and open only if a lower quota of participants is reached.

When preferences of the agents are taken into account for selecting the projects to assign, a naive strategy is to first select the projects via a multiwinner voting rule and then allocate the projects. Nevertheless, this approach may lead to select projects that do not fit any agent personally, like in the conference example. Conversely, by only considering allocation purposes, one could impose conference days where only a few attendants can be present. Our model can be seen as a particular case of house allocation with externalities where agents care about their assignment and the set of selected projects (but not about the identity of the owners). In the notion of envy-freeness conditioned to the approval of the majority of agents [Shams et al., 2020], agents can also give their opinion about the assignment of other agents, but there is no concern of the set of selected projects. The goal of our article is to find a reasonable way to combine both allocation and selection goals, by focusing on fairness and optimality issues.

\section{Allocation of Projects}

We consider a set of agents $N=[n]:=\{1, \ldots, n\}$ and a set of projects $P=\left\{p_{1}, \ldots, p_{m}\right\}$ with $m \geq n$. Each agent $i$ has ordinal preferences over projects, represented by a linear order $\succ_{i}$ over $P$. A feasible allocation $\sigma: N \rightarrow P$ is a matching, perfect w.r.t. the agents, where every agent is assigned to exactly one project and no two agents share the same project, i.e., $\sigma(i) \neq \sigma(j)$ for every agents $i \neq j$ and $\sigma(i) \in P$ denotes the project assigned to agent $i$ in allocation $\sigma$. Typically, some projects may not be assigned. Let $P_{\sigma}$ denote the set of assigned projects in allocation $\sigma$, i.e., $P_{\sigma}:=$ $\{p \in P: \exists i \in N$ such that $\sigma(i)=p\}$. An instance of the selection-allocation (SA) problem is $I=\left(N, P,\left(\succ_{i}\right)_{i \in N}\right){ }^{1}$

\subsection{Finding an Optimal and Fair Allocation}

An allocation $\sigma$ is Pareto-optimal (PO) if there is no feasible allocation $\sigma^{\prime}$ such that $\sigma^{\prime}(i) \succeq_{i} \sigma(i)$ for every agent $i$ and there exists an agent $j$ such that $\sigma^{\prime}(j) \succ_{j} \sigma(j)$. In an ordinal setting, PO can be refined with rank-maximality $(R M)$. The signature $\rho_{\sigma}$ of allocation $\sigma$ is the $m$-vector giving the number of agents $\rho_{\sigma}(k)$ assigned to their $k^{\text {th }}$ most preferred project in $\sigma$, for all $k \in[m]$. Signature $\rho_{\sigma}$ is lexicographically strictly greater than signature $\rho_{\sigma^{\prime}}$, denoted by $\rho_{\sigma}>_{\text {lex }} \rho_{\sigma^{\prime}}$, if

\footnotetext{
${ }^{1}$ The problem is similar to house allocation but we use a different name to emphasize the combined goal of selection and allocation.
}

there exists an index $i \in[m]$ such that $\rho_{\sigma}\left(i^{\prime}\right)=\rho_{\sigma^{\prime}}\left(i^{\prime}\right)$ for all $i^{\prime}<i$ and $\rho_{\sigma}(i)>\rho_{\sigma^{\prime}}(i)$. An RM allocation can be computed in polynomial time [Irving et al., 2006].

Definition 1 (Rank-maximality (RM)). An allocation $\sigma$ is $R M$ if there is no feasible allocation $\sigma^{\prime}$ such that $\rho_{\sigma^{\prime}}>_{\text {lex }} \rho_{\sigma}$.

Popularity is another optimality measure that refines PO. Agent $i$ prefers allocation $\sigma$ to allocation $\sigma^{\prime}$ if $\sigma(i) \succ_{i} \sigma^{\prime}(i)$. An allocation $\sigma$ is then more popular than another allocation $\sigma^{\prime}$ if the number of agents who prefer $\sigma$ to $\sigma^{\prime}$ is strictly greater than the number of agents who prefer $\sigma^{\prime}$ to $\sigma$.

Definition 2 (Popularity (Pop)). An allocation $\sigma$ is popular if there is no feasible allocation $\sigma^{\prime}$ more popular than $\sigma$.

A Pop allocation may not exist but existence can be efficiently checked and, in the positive case, a Pop allocation can be computed in polynomial time, thanks to the following characterization due to Abraham et al. [2007]: An allocation $\sigma$ is Pop iff (1) every first-ranked project is assigned in $\sigma$, and (2) $\sigma$ assigns to every agent either her first-ranked project or the most preferred project that is not ranked first by an agent.

Fairness issues can also be considered. We relax envyfreeness with rank-envy-freeness, where an agent $i$ is envious towards an agent $j$ only if agent $i$ ranks the envied project in a better position in her preference ranking than agent $j$. Let $r_{i}: P \rightarrow[m]$ be the function giving the rank of a project in $\succ_{i}$, i.e., $r_{i}(x)=\left|\left\{y \in P: y \succeq_{i} x\right\}\right|$, for project $x \in P$.

Definition 3 (Rank-envy-freeness (r-EF)). Allocation $\sigma$ is $r$ EF if $\sigma(i) \succ_{i} \sigma(j)$ or $r_{j}(\sigma(j)) \leq r_{i}(\sigma(j))$ for every $i, j \in N$.

A rank-maximal or popular allocation is also r-EF.

Proposition 1. $R M \Rightarrow r-E F$ and Pop $\Rightarrow r-E F$.

Proof. Suppose that an RM allocation $\sigma$ is not r-EF. There exist agents $i$ and $j$ such that $\sigma(j) \succ_{i} \sigma(i)$ and $r_{i}(\sigma(j))<$ $r_{j}(\sigma(j))$. Hence, both $r_{i}(\sigma(j))<r_{j}(\sigma(j))$ and $r_{i}(\sigma(j))<$ $r_{i}(\sigma(i))$ hold. Thus, by swapping the projects of $i$ and $j$, we get an allocation $\sigma^{\prime}$ such that $\rho_{\sigma^{\prime}}>_{\text {lex }} \rho_{\sigma}$, a contradiction.

Suppose that a Pop allocation $\sigma$ is not r-EF. There exist agents $i$ and $j$ such that $\sigma(j) \succ_{i} \sigma(i)$ and $r_{i}(\sigma(j))<$ $r_{j}(\sigma(j))$. By rank-envy, neither agent $i$ nor agent $j$ is assigned her first-ranked project in $\sigma$ so, by Abraham et al. [2007]'s characterization, they are both assigned her most preferred project that is not ranked first by an agent. Since $i$ prefers $\sigma(j)$ to $\sigma(i)$, project $\sigma(j)$ must be ranked first by some agent, contradicting the fact that agent $j$ is assigned her most preferred project that is not ranked first by any agent.

The reverse of both implications is not true, since r-EF does not even imply PO. Moreover, although both RM and Pop imply r-EF, it is not true for their weaker requirement PO.

Example 1. For $r-E F \nRightarrow P O$, simply consider an instance with two agents and three projects with the following preferences: $p_{1} \succ_{1} p_{2} \succ_{1} p_{3}$ and $p_{1} \succ_{2} p_{3} \succ_{2} p_{2}$. Allocation assigning $p_{2}$ to agent 1 and $p_{3}$ to agent 2 is $r$-EF but not $P O$.

For $P O \nRightarrow r-E F$, consider an instance with three agents and three projects. The preferences are given below.

$$
\begin{aligned}
& 1: p_{1} \succ p_{3} \succ p_{2} \\
& 2: p_{2} \succ p_{1} \succ p_{3} \\
& 3: \quad p_{2} \succ p_{1} \succ p_{3}
\end{aligned}
$$


The encircled allocation is PO but not r-EF: agent 1 is envious towards agent 2 who owns her second ranked project $p_{1}$ which is the first-ranked project of agent 1 .

For rank $0 \leq k<m$, we define a generalization of r-EF.

Definition $4\left(\right.$ Rank $_{k}$-envy-freeness $\left.\left(\mathrm{r}_{k}-\mathrm{EF}\right)\right)$. An allocation $\sigma$ is rank $k_{k}$-envy-free if for every agents $i$ and $j, \sigma(i) \succ_{i} \sigma(j)$ or $r_{j}(\sigma(j)) \leq \min \left\{r_{i}(\sigma(j)), k\right\}$ holds.

Note that $\mathrm{r}_{k}-\mathrm{EF} \Rightarrow \mathrm{r}_{k^{\prime}}-\mathrm{EF}$ for every $k^{\prime}>k$. Moreover, $\mathrm{r}_{m-1}-\mathrm{EF} \Leftrightarrow \mathrm{r}-\mathrm{EF}$ and $\mathrm{r}_{0}$-EF $\Leftrightarrow$ envy-freeness $(\mathrm{EF})$. By the fact that $\mathrm{RM} \Rightarrow \mathrm{r}-\mathrm{EF}$, an $\mathrm{r}_{m-1}$-EF allocation always exists. However, starting from $k=m-2$, an $\mathrm{r}_{k}$-EF allocation may not exist, as shown in the basic example with three agents and three projects where every agent has the same preferences.

\subsection{Towards a Good Selection}

We introduce a simple requirement imposing that no agent prefers a project that has not even been assigned.

Definition 5 (Frustration-freeness (FF)). An allocation $\sigma$ is FF if $\sigma(i) \succ_{i}$ p for every agent $i$ and every project $p \in P \backslash P_{\sigma}$.

When $m=n$, all projects are selected so every allocation is FF. Frustration is defined according to selected projects, so it does not say anything about global optimality. In particular, it is clear that an FF allocation may not be PO. However, conversely, in every SA instance, a PO allocation (and thus an RM or a Pop allocation) is FF: otherwise by assigning the preferred unassigned project to the agent who prefers it over her current assigned project, we get an allocation that Paretodominates it. Therefore, an FF allocation always exists.

The issues forbidding r-EF or FF to imply Paretooptimality can be fixed by combining the two properties.

Proposition 2. $r-E F+F F \Rightarrow P O$.

Proof. Consider an allocation $\sigma$ that is r-EF and FF. Take an allocation $\sigma^{\prime}$ that Pareto-dominates $\sigma$. For every agent $i$, either $\sigma^{\prime}(i)=\sigma(i)$, or $\sigma^{\prime}(i) \succ_{i} \sigma(i)$. So, by FF, we have $P_{\sigma}=$ $P_{\sigma^{\prime}}$. Consider an ameliorating cycle $\left(i_{0}, i_{1}, \ldots, i_{c-1}\right) \subseteq N$ from $\sigma$ to $\sigma^{\prime}$, i.e., $\sigma^{\prime}\left(i_{j-1}\right)=\sigma\left(i_{j} \bmod c\right)$ for every $j \in[c]$. Since $\sigma^{\prime}\left(i_{j-1}\right)=\sigma\left(i_{j} \bmod c\right) \succ_{i_{j-1}} \sigma\left(i_{j-1}\right)$, by r-EF we must have $r_{i_{j} \bmod c}\left(\sigma\left(i_{j} \bmod c\right)\right) \leq r_{i_{j-1}}\left(\sigma\left(i_{j} \bmod c\right)\right)<$ $r_{i_{j-1}}\left(\sigma\left(i_{j-1}\right)\right)$, for every $j \in[c]$, a contradiction.

Combining FF with $\mathrm{r}_{1}$-EF provides an alternative characterization of Pop. An agent $i$ is $\mathbf{r}_{1}$-envious towards an agent $j$ only if the envied project is not ranked first by agent $j$.

Theorem 1. For every SA instance, Pop $\Leftrightarrow r_{1}-E F+F F$.

\section{Hard Constraints over the Selected Projects}

In this section, we assume that the set of projects is partitioned into $q$ groups, i.e., $P=P_{1} \cup \cdots \cup P_{q}$, and that a feasible allocation must satisfy some quotas on the selected projects within groups. Let $\ell_{j}$ and $u_{j}$ denote the respective lower and upper quotas for group $P_{j}$ for $j \in[q]$; trivially we assume that $\ell_{j} \leq u_{j} \leq\left|P_{j}\right|$ for every $j \in[q]$. An allocation $\sigma$ is feasible if $\ell_{j} \leq\left|\bar{P}_{\sigma} \cap P_{j}\right| \leq u_{j}$ for every $j \in[q]$. We suppose that $\sum_{j \in[q]} \ell_{j} \leq n \leq \sum_{j \in[q]} u_{j}$, otherwise there is no feasible allocation. An instance of this constrained selection-allocation (CSA) problem is $\left(N, P,\left(\succ_{i}\right)_{i \in N},\left(P_{j}\right)_{j \in[q]},\left(\ell_{j}, u_{j}\right)_{j \in[q]}\right)$.
An SA instance is a CSA instance where $\ell_{j}=0$ and $u_{j}=\left|P_{j}\right|$ for every $j \in[q]$. An instance is said with no trivial group upper quota if $u_{j}<\left|P_{j}\right|$ for every $j \in[q]$. Let $\succ_{i}^{P_{j}}$ be the preference ranking of agent $i$ within group $P_{j}$. Preferences are group-oriented if every agent $i$ 's preferences can be decomposed w.r.t. project groups, i.e., there is a permutation $\tau_{i}:[q] \rightarrow[q]$ such that for every projects $p \in P_{j}$ and $p^{\prime} \in P_{j^{\prime}}$, $p \succ_{i} p^{\prime}$ iff $p \succ_{i}^{P_{j}} p^{\prime}$ if $j=j^{\prime}$ or $\tau_{i}(j)<\tau_{i}\left(j^{\prime}\right)$ otherwise.

Contrary to the SA setting, even an RM and Pop allocation may not be FF in a CSA instance, because of the constraints on project groups, even if an FF allocation exists.

Example 2. Let us consider a CSA instance with four agents and five projects where $P_{1}=\left\{p_{1}, p_{3}, p_{4}\right\}, P_{2}=\left\{p_{2}, p_{5}\right\}$, and $u_{1}=2$. The preferences are given below.

$$
\begin{aligned}
& 1: p_{3} \succ p_{1} \succ p_{4} \succ p_{2} \succ p_{5} \\
& 2: p_{3} \succ p_{1} \succ p_{4} \succ p_{2} \succ p_{5} \\
& 3: p_{1} \succ p_{3} \succ p_{2} \succ p_{4} \succ p_{5} \\
& 4: p_{2} \succ p_{5} \succ p_{1} \succ p_{3} \succ p_{4}
\end{aligned}
$$

The framed allocation is $R M$ with signature $(3,0,0,0,1)$ and Pop. However, it is not FF since agent 1 is frustrated: she prefers the unassigned project $p_{4}$. Nevertheless, the encircled allocation, with signature $(1,2,1,0,0)$, is both $P O$ and $F F$.

It follows that the characterization obtained in Theorem 1 does not hold in a CSA instance. By replacing FF by PO, we can nevertheless keep the validity of necessary conditions.

Proposition 3. In every CSA instance, Pop $\Rightarrow r_{1}-E F+P O$.

Proof. A Pop allocation $\sigma$ is trivially PO. Suppose that $\sigma$ is not $\mathrm{r}_{1}$-EF. There exist agents $i$ and $j$ such that $\sigma(j) \succ_{i} \sigma(i)$ and $r_{j}(\sigma(j))>1$. Thus, $j$ does not rank $\sigma(j)$ first. If there exists a project $p \in P_{\sigma}$ assigned to an agent $k$ such that $p \succ_{j} \sigma(j)$ then, by assigning project $\sigma(j)$ to $i$, project $p$ to $j$ and project $\sigma(i)$ to $k$, we reach an allocation more popular than $\sigma$ ( $i$ and $j$ are better off and $k$ may be worse off), a contradiction. Otherwise, there exists a project $p \in P \backslash P_{\sigma}$ such that $p \succ_{j} \sigma(j)$. If $p$ cannot be assigned, it means that it belongs to a group $P_{\ell}$ whose upper quota $u_{\ell}$ is reached. By removing from $P_{\sigma}$ an arbitrary project belonging to $P_{\ell} \backslash\{\sigma(j)\}$ that was assigned to an agent $k$, we can assign $p$ to $j, \sigma(j)$ to $i$ and an arbitrary project respecting the constraints to $k$. This new allocation is more popular than $\sigma(i$ and $j$ are better off and $k$ may be worse off), a contradiction. If there is no assigned project belonging to $P_{\ell}$ different from $\sigma(j)$, then $u_{\ell}=1$ and $P_{\sigma} \cap P_{\ell}=\{\sigma(j)\}$. Hence, $\sigma(i) \notin P_{\ell}$ and reassigning project $p$ to $j$ instead of $\sigma(j)$ is feasible and construct an allocation more popular than $\sigma$, a contradiction.

However, $\mathrm{r}_{1}-\mathrm{EF}+\mathrm{PO} \nRightarrow$ Pop, as illustrated below.

Example 3. Let us consider a CSA instance with three agents and four projects where $P_{1}=\left\{p_{1}, p_{4}\right\}, P_{2}=\left\{p_{2}, p_{3}\right\}$ and $u_{1}=1$. The preferences are given below.

$$
\begin{aligned}
& 1: p_{1} \succ p_{3} \succ p_{2} \succ p_{4} \\
& 2: p_{2} \succ p_{3} \succ p_{1} \succ p_{4} \\
& 3: p_{2} \succ p_{4} \succ p_{1} \succ p_{3}
\end{aligned}
$$

The encircled allocation is $P O$ and $r_{1}-E F$ but not popular. The framed allocation is both rank-maximal and popular. 
In Example 2, the intersection between the set of RM or Pop allocations and the set of FF allocations is empty. However, this does not hold for the weaker requirement PO. If an FF allocation exists, then an allocation that is both $\mathrm{PO}$ and FF exists: it suffices to start a top-trading cycle algorithm [Shapley and Scarf, 1974] from an FF allocation. In general, an FF allocation may not exist: consider an instance with two agents where their first-ranked projects are $p_{1}$ and $p_{2}$, respectively, which belong to the same group with upper quota one. Moreover, deciding the existence of FF allocations is hard.

Theorem 2. It is NP-complete to decide whether there exists an FF allocation in a CSA instance, even either under grouporiented preferences or with no trivial group upper quota.

In Theorem 2's reduction, preferences are group-oriented or there is no trivial group upper quota, but not both simultaneously. Indeed, under both restrictions, one can decide in polynomial time about the existence of an FF allocation.

Proposition 4. The existence of an FF allocation can be decided in polynomial time in every CSA instance with no trivial group upper quota under group-oriented preferences. If existence is guaranteed, one can then construct an FF and RM (or Pop if exists) allocation in polynomial time.

Sketch of proof. Let $N_{j}$ denote the set of agents who prefer the most the projects in $P_{j}$. One can show that, under group-oriented preferences when $u_{j}<\left|P_{j}\right|$ for every $j \in[q]$, there exists an FF allocation iff $\ell_{j} \leq\left|N_{j}\right| \leq u_{j}$ for every $j \in[q]$. Under such conditions, each FF allocation or each PO allocation assigns to each agent in $N_{j}$ a project in $P_{j}$. Thus, each PO allocation is FF and is the concatenation of PO allocations in all SA sub-instances $\left(N_{j}, P_{j},\left(\succ_{i}\right)_{i \in N_{j}}\right)_{j \in[q]}$. Recall that in each SA sub-instance, an RM allocation or Pop allocation (if exists) can be found in polynomial time.

\section{Selecting Socially Good Projects}

In this section, we aim to assign projects to the agents such that the whole set of selected projects is "good" according to the preferences of the agents. We evaluate the quality of projects w.r.t. pairwise comparisons. Let $N\left(p, p^{\prime}\right)$ denote the set of agents preferring project $p$ to project $p^{\prime}$. A project $p$ is said to dominate another project $p^{\prime}$ if $\left|N\left(p, p^{\prime}\right)\right|>n / 2$. The set of projects that dominate project $p$ is denoted by $D(p)$. In a voting setting, when focusing on pairwise comparisons, it is highly desirable to select a Condorcet winner alternative which dominates all the others, and to not select a Condorcet loser alternative which is dominated by all the others. We adapt these requirements to the context of allocation.

Definition 6 (Condorcet $\{$ winner / loser $\}$ criterion ( $\{\mathrm{CWC} /$ $\mathrm{CLC}\}))$. Allocation $\sigma$ satisfies $C W C$ if, when there exists a Condorcet winner project $p$ then project $p$ is assigned in $\sigma$. Allocation $\sigma$ satisfies CLC if, when $m>n$ and there exists a Condorcet loser project $p$ then project $p$ is not assigned in $\sigma$.

There is already a conflict between selection and allocation desiderata since the two Condorcet axioms for selection can be incompatible with FF (and thus PO, RM and Pop).

Proposition 5. CWC or CLC is incompatible with FF.
Proof. Let us consider an instance with $n>2$ agents where the preferences are given below.

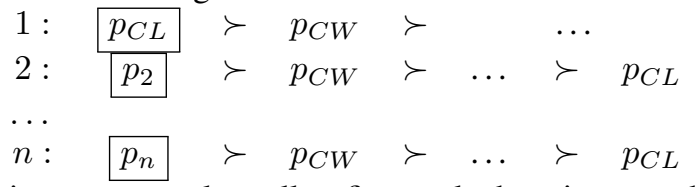

FF imposes to select all $n$ first-ranked projects, and thus Condorcet loser $p_{C L}$ but not Condorcet winner $p_{C W}$.

Preferences $\succ$ over single projects can be extended to sets of projects by using a preference extension $\succ^{e x t}$. A preference extension $\succ^{\text {ext }}$ satisfies responsiveness [Roth, 1985; Barberà et al., 2004] if $\forall x \in X \subset P$ and $\forall y \in P \backslash X$, $(X \backslash\{x\}) \cup\{y\} \succ^{e x t} X$ holds iff $y \succ x$ holds. The responsive set extension $\succ^{R S}$ is the minimal partial order that satisfies responsiveness. We also consider extension $\succ^{R S^{-}}$, a weaker version of $\succ^{R S}$ where transitivity is dropped. Although the latter notion may appear unnatural for modelling preferences, in the context of an SA instance, it means that if two sets of selected projects are too different then only the quality of the allocation matters. When referring to a given preference extension $\succ^{e x t}$, we assume that each agent $i$ uses $\succ_{i}^{e x t}$.

We derive a variant of popularity for taking into account the preferences of the agents over selected projects. Let $n_{1}\left(\sigma^{\prime}, \sigma\right)$ denote the number of agents who get a better assignment in $\sigma^{\prime}$ than in $\sigma$, and $n_{2}^{\succ^{e x t}}\left(\sigma^{\prime}, \sigma\right)$ the number of agents who get the same assignment but prefer set $P_{\sigma^{\prime}}$ to $P_{\sigma}$ w.r.t. $\succ^{e x t}$. Let $n_{\succ \text { ext }}^{\text {Pop }}\left(\sigma^{\prime}, \sigma\right)$ denote the number of agents who prefer allocation $\sigma^{\prime}$ to $\sigma$ in the sense that they prefer to get a better assigned project and if they keep the same project, they consider the selected projects, i.e., $n_{\succ \text { ext }}^{\text {Pop }}\left(\sigma^{\prime}, \sigma\right)=n_{1}\left(\sigma^{\prime}, \sigma\right)+$ $n_{2}^{\succ^{e x t}}\left(\sigma^{\prime}, \sigma\right)$. Allocation $\sigma$ is more popular w.r.t. $\succ^{e x t}$ than allocation $\sigma^{\prime}$ if $n_{\succ \text { ext }}^{\text {Pop }}\left(\sigma^{\prime}, \sigma\right)>n_{\succ \text { ext }}^{\text {Pop }}\left(\sigma, \sigma^{\prime}\right)$. This notion generalizes standard popularity to an SA instance with externalities where agents care about the other assigned projects.

Definition 7 (Popularity w.r.t. $\left.\succ^{\text {ext }}\left(\mathrm{Pop}_{\succ} \mathrm{ext}\right)\right)$. An allocation $\sigma$ is Pop $_{\succ}$ ext if there is no other feasible allocation $\sigma^{\prime}$ that is more popular w.r.t. preference extension $\succ^{e x t}$.

A Pop $\succ$ ext allocation may not be Pop and vice versa, as shown in Example 4. However, when $n=m$ the two notions coincide, showing that a Pop $\succ$ ext allocation may not exist.

An allocation $\sigma$ satisfies dominion selection $(D S)$ if $\bigcup_{p \in P_{\sigma}} D(p) \subseteq P_{\sigma}$. We show that, for any responsive $\succ^{e x t}$, Pop $_{\succ}$ ext satisfies both dominion selection and $r_{1}$-EF restricted to the set of selected projects, called $\mathrm{r}_{1}^{P_{\sigma}}$-EF, where there is no pair of agents $i$ and $j$ such that $i$ prefers the project allocated to $j$ but this project is not ranked first by $j$ within $P_{\sigma}$.

Proposition 6. If $\succ^{e x t}$ is responsive, $P_{o p^{e x t}} \Rightarrow D S+r_{1}^{P_{\sigma}}-E F$.

Proof. Consider a Pop $\operatorname{Pext}_{\text {ext }}$ allocation $\sigma$. Suppose that $\sigma$ does not satisfy DS. Then, there exists a project $p \in P_{\sigma}$ dominated by $p^{\prime} \in P \backslash P_{\sigma}$. Consider the allocation $\sigma^{\prime}$ constructed from $\sigma$ where agent $i$ who was assigned $p$ in $\sigma$ is assigned $p^{\prime}$. By definition of dominance, at least $\left\lceil\frac{n+1}{2}\right\rceil$ agents prefer $\sigma^{\prime}$ to $\sigma$, a contradiction. Suppose now that $\sigma$ is not $\mathrm{r}_{1}^{P_{\sigma}}$-EF. There exist agents $i$ and $j$ and project $p \in P_{\sigma}$ such that $\sigma(j) \succ_{i} \sigma(i)$ 
and $p \succ_{j} \sigma(j)$. Consider the allocation $\sigma^{\prime}$ constructed from $\sigma$ where agent $i$ is assigned $\sigma(j)$, agent $j$ is assigned $p$ and agent $k$ who was possessing $p$ is assigned $\sigma(i)$. Since $P_{\sigma}=P_{\sigma^{\prime}}$, we only need to consider the satisfaction of agents $i, j$ and $k$, and two of them ( $i$ and $j$ ) are better off in $\sigma^{\prime}$, a contradiction.

From the satisfaction of DS, when $m>n$, a Pop $\succ$ ext allocation cannot select a Condorcet loser $p$, for a responsive $\succ^{e x t}$, since $p$ is dominated by at least $n$ other projects. Moreover, a project that dominates at least $m-n$ other projects must be selected since at least one of its dominated projects is selected. Hence, the Condorcet winner is selected.

Corollary 1. If $\succ^{\text {ext }}$ is responsive, $P o p_{\succ}$ ext $\Rightarrow C W C+C L C$.

Contrary to standard popularity, as expected from Proposition 5, if $\succ^{e x t}$ is responsive, then $\mathrm{Pop}_{\succ \text { ext }}$ fails FF. However, from Proposition 6, it satisfies a mild relaxation of FF, imposing to an allocation $\sigma$ that if there exists a project $p \in P \backslash P_{\sigma}$ and an agent $i$ such that $p \succ_{i} \sigma(i)$, then $p$ cannot dominate any project in $P_{\sigma}$. Nevertheless, even $\mathrm{Pop}_{\succ}$ Rs may fail r-EF.

Example 4. Let us consider an SA instance with five agents and six projects where the preferences are given below.

$$
\begin{aligned}
& 1: p_{1} \succ p_{5} \succ p_{3} \succ p_{4} \succ p_{2} \succ \underline{p_{6}} \\
& 2: \underline{\underline{p_{5}}} \succ p_{1} \succ p_{3} \succ p_{4} \succ p_{2} \succ \overline{p_{6}} \\
& 3: \quad p_{2} \succ p_{3} \succ p_{4} \succ p_{5} \succ p_{6} \succ p_{1} \\
& 4: p_{3} \succ p_{2} \succ p_{4} \succ p_{5} \succ p_{6} \succ p_{1} \\
& 5: \quad p_{4} \succ p_{2} \succ p_{3} \succ p_{5} \succ p_{6} \succ p_{1}
\end{aligned}
$$

The underlined and encircled allocations are $P_{o p} p_{\succ s}$ but the latter is not r-EF. The only Pop allocation is framed.

In case of r-EF failure, $r-E F$ swaps can be performed: agents $i$ and $j$ can swap their projects if $i$ is rank-envious towards $j$. We show that r-EF swaps preserve $\mathrm{Pop}_{\succ}$ ext.

Proposition 7. For every $\succ^{\text {ext }}$, if a Pop $\succ$ ext allocation $\sigma$ exists, then an allocation that is both Pop $_{\succ}$ ext and $r$-EF can be reached in polynomial time via $r$-EF swaps starting from $\sigma$.

Proof. Consider a Pop $\succ$ ext allocation $\sigma$ that is not r-EF. There exist two agents $i$ and $j$ such that $i$ is rank-envious towards $j$. Let $\sigma^{\prime}$ denote the allocation resulting from an r-EF swap between $i$ and $j$ in $\sigma$. We have $P_{\sigma}=P_{\sigma^{\prime}}$, so $n_{2}^{\succ^{e x t}}\left(\sigma^{\prime}, \sigma\right)=$ $n_{2}^{\succ^{e x t}}\left(\sigma, \sigma^{\prime}\right)=0$. By rank-envy, $\sigma^{\prime}(i) \succ_{i} \sigma(i)$. Thus, $\sigma(j) \succ_{j} \sigma(i)$, otherwise $i$ and $j$ would be $\mathrm{r}_{1}^{P_{\sigma}}$-envious in $\sigma$. Hence, $\sigma^{\prime}$ is as popular w.r.t. $\succ^{e x t}$ as $\sigma$. By $\mathrm{r}_{1}^{P_{\sigma}}$-EF and DS, if an allocation is more popular w.r.t. $\succ^{e x t}$ than $\sigma^{\prime}$, then it is also true for $\sigma$, a contradiction. Hence, $\sigma^{\prime}$ is also $\mathrm{Pop}_{\succ \text { ext }}$.

A Pop $\succ$ ext allocation $\sigma$ is Pop when $P=P_{\sigma}$ and the number of Pop allocations is polynomial [McDermid and Irving, 2011]. Since r-EF swaps transform a $\operatorname{Pop}_{\succ}$ ext allocation $\sigma$ to another $\mathrm{Pop}_{\succ \text { ext }}$ allocation $\sigma^{\prime}$ where $P_{\sigma^{\prime}}=P_{\sigma}$ but with a strictly greater signature w.r.t. $>_{l e x}$, the dynamics of r-EF swaps starting from $\sigma$ converges in polynomial time.

However, although $\mathrm{Pop}_{\succ} \mathrm{RS}$ is attractive and despite the fact that a popular allocation can be computed in polynomial time, even the verification of a $\operatorname{Pop}_{\succ} \mathrm{RS}$ allocation is hard.

Theorem 3. Checking popularity w.r.t. $\succ^{R S}$ is co-NP-hard.
The complexity proof mostly relies on set comparisons induced by $\succ^{R S}$. However, an allocation is not $\mathrm{Pop}_{\succ \mathrm{RS}}$ if there exists an unselected project $p$ that is preferred to a selected project $p^{\prime}$ by a "blocking" subset of agents and the remaining subset of agents can be reassigned such that there are more, or as many, better off agents than worse off agents. Hence, we conjecture that checking Pop $\mathrm{P}_{\succ S^{-}}$is also hard.

To circumvent the problem of a blocking subset of agents, we derive another variant of popularity that takes into account the preferences of all agents over selected projects. For preference extension $\succ^{e x t}$, we denote by $n_{3}^{\succ^{e x t}}\left(\sigma^{\prime}, \sigma\right)$ the number of agents who prefer $P_{\sigma^{\prime}}$ to $P_{\sigma}$, and define $n_{\succ \text { ext }}^{2 P_{\text {op }}}\left(\sigma^{\prime}, \sigma\right):=$ $n_{1}\left(\sigma^{\prime}, \sigma\right)+n_{3}^{\succ^{e x t}}\left(\sigma^{\prime}, \sigma\right)$. Allocation $\sigma^{\prime}$ is more 2popular w.r.t. $\succ^{\text {ext }}$ than allocation $\sigma$ if $n_{\succ \text { ext }}^{2 P^{\text {exp }}}\left(\sigma^{\prime}, \sigma\right)>n_{\succ \text { ext }}^{2 \text { Pop }^{2}}\left(\sigma, \sigma^{\prime}\right)$.

Definition 8 (2Pop $\succ e x t)$. Allocation $\sigma$ is (strictly) 2popular w.r.t. $\succ^{\text {ext }}$ if there is no other allocation that is more 2popular (at least as 2popular) w.r.t. $\succ^{\text {ext }}$.

$2 \mathrm{Pop}_{\succ \text { ext }}$ allocations always select a Condorcet winner and strictly $2 \mathrm{Pop}_{\succ \text { ext }}$ allocations never select a Condorcet loser.

Proposition 8. If $\succ^{e x t}$ is responsive, $2 P_{\succ o p} p_{\text {ext }} \Rightarrow C W C$ and strict 2 Pop $_{\succ \text { ext }} \Rightarrow$ CLC.

Moreover, by the same proof idea as Proposition 7, for every $\succ^{e x t}$, if a $2 \mathrm{Pop}_{\succ}{ }^{e x t}$ exists then $\mathrm{r}$-EF swaps can reach in polynomial time an allocation that is both $2 \mathrm{Pop}_{\succ}$ ext and $\mathrm{r}-\mathrm{EF}$.

By slightly adapting the proof of Theorem 3, even the verification of $2 \mathrm{Pop}_{\succ} \mathrm{R}_{S}$ is co-NP-hard. We consequently focus on the weaker version of $\succ^{R S}$ where transitivity is dropped. Note that $2 \mathrm{Pop}_{\succ R S^{-}}$can be characterized based on $\mathrm{r}_{1}^{P_{\sigma}}$-EF, a refinement of FF and dominance conditions w.r.t. unassigned projects. These conditions can be verified in polynomial time.

Theorem 4. $2 \mathrm{Pop}_{\succ} \mathrm{RS}^{-}$can be verified in polynomial time.

The question of computation for $2 \mathrm{Pop}_{\succ} \mathrm{SS}^{-}$remains open.

\section{Empirical Evaluation of Allocations}

In this section, we empirically evaluate the quality of different procedures for assigning projects to agents, both according to selection and allocation considerations. We generate $100 \mathrm{SA}$ instances with $n=5$ agents and $m=15$ projects. Correlated preferences are studied via the single-peaked preference domain. A preference ranking $\succ_{i}$ is single-peaked if there exists a linear order $>$ over $P$ such that for all projects $x, y, z$ with $x>y>z$ or $z>y>x, x \succ_{i} y$ implies $y \succ_{i} z$. We consider three types of preference generation: impartial culture $(I C)$ where each preference ranking is uniformly drawn from the set of all possible preference rankings, single-peaked uniform peak (SP-UP) where each single-peaked preference ranking is generated by first uniformly selecting the peak project then uniformly choosing the next ranked project either on the left of the peak in axis $>$ or on the right and so on [Conitzer, 2009], and single-peaked uniform (SP-U) where each preference ranking is uniformly drawn from the set of all possible single-peaked rankings [Walsh, 2015]. Under SP-U and SP-UP, preferences are correlated w.r.t. a common axis > along projects. Under SP-UP, a preference ranking with, as a first-ranked project, an extreme point of the axis has the same 


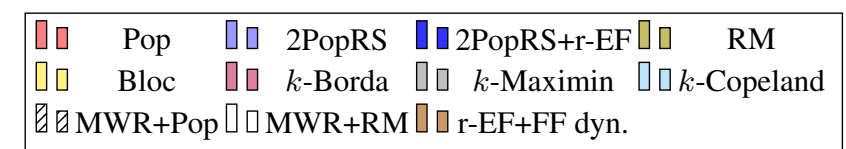
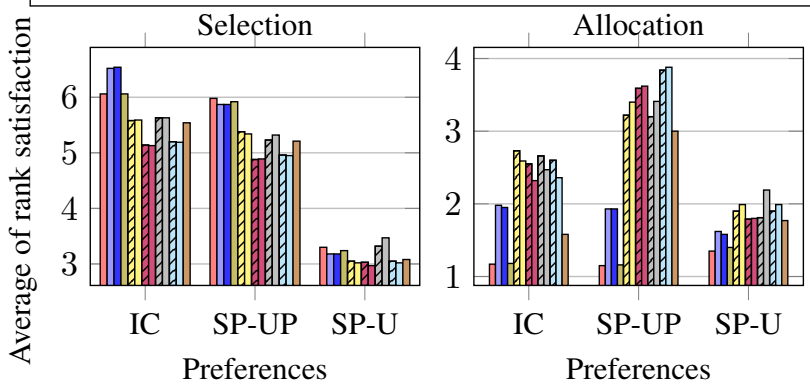

Figure 1: Average over 100 runs of the rank satisfaction for different types of allocations when evaluating the selection (left) or the allocation (right) for $n=5$ and $m=15$

probability to occur as one with a middle point of the axis. Hence, the generated preferences are more diverse under SPUP than under SP-U, where the occurrence of a project at a certain preference rank depends on a binomial coefficient.

For evaluating the quality of the set of selected projects (selection goal), we compute the satisfaction of an agent by considering the average rank in her preference ranking over all selected projects. The global rank satisfaction is then computed by averaging all individual average rank satisfactions. For evaluating the quality of the allocation (allocation goal), we consider the average over all agents of the rank given to their allocated project. Our results are given in Figure 1.

All possible allocations are generated and the measures are given in average for different types of allocations:

- Optimal allocations: Pop allocations or RM allocations;

- Selection-based optimal (Sel-opt) allocations: a multiwinner voting rule (MWR) is used for selecting $n$ projects, and then a Pop or an RM allocation is computed on an SA instance where projects are restricted to this set. We use the following excellence-based MWRs: Bloc, $k$-Borda, $k$-Maximin, and $k$ Copeland (see Faliszewski et al. [2017] for definitions).

- Selection \& allocation (SA)-based allocations: $2 \mathrm{Pop}_{\succ R S^{-}}$ allocations (2PopRS), 2Pop $\mathrm{P} S^{-}$allocations that are $\mathrm{r}-\mathrm{EF}$, and allocations (r-EF+FF dyn.) which are the result of the dynamics of r-EF swaps and FF-agreement moves (an agent $i$ can get a more preferred unassigned project $p$ if the majority of agents prefers $p$ to $i$ 's project) from a random allocation.

As expected, optimal allocations behave significantly better under an allocation goal than Sel-opt allocations and reversely under a selection goal. Our variant of Pop, $2 \mathrm{Pop}_{\succ R S^{-}}$, is not satisfactory under IC where the average rank satisfactions are always superior than those of Pop or RM. However, it performs a bit better with a selection goal when preferences are correlated. Indeed, under single-peaked distributions, the satisfaction of Condorcet criteria should help to choose a better set of selected projects. Surprisingly, the stable allocation arising from the dynamics associated with r-EF swaps and FF-agreement moves appears to be very good for combining both goals of selection and allocation. It has an intermediate behavior with an average rank satisfaction closer to those of Sel-opt allocations than optimal allocations with a selection goal, and closer to optimal allocations than Sel-opt allocations with an allocation goal. In average, ten steps are sufficient to reach a stable allocation so we are far from the exponential theoretical bound for r-EF swaps.

So far, we have evaluated the quality of an allocation (allocation goal) via the optimality measure of average rank satisfaction. For fairness, we compute the average minimum $k$ such that an allocation is $\mathrm{r}_{k}$-EF. Our experiments show that optimal allocations and SA-based allocations are very fair. However, Sel-opt allocations are very unfair in comparison, showing the need of combining allocation and selection goals. In general, a slight tendency confirms the intuition under which correlated preferences favor consensus in selection whereas diverse preferences favor consensus in allocation.

\section{Conclusion}

We investigated a variant of resource allocation where both selection and allocation are important. We focused on popularity (Pop) and rank-maximality (RM) optimality concepts and showed that they are compatible with two notions of fairness that we have introduced or refined, namely frustrationfreeness (FF) and rank-envy-freeness (r-EF). In an ordinal context, r-EF appears as a very natural and flexible fairness requirement that could easily be used as an argument for explaining a decision. The selection goal has been combined with the allocation goal in two ways: via hard constraints represented by quotas on project groups or via the preferences of the agents themselves. Under hard constraints, RM and Pop lose their link with FF and this latter basic requirement even becomes difficult to satisfy. However, we identified natural restrictions under which the compatibility remains. When the selection goal is conditioned by the preferences of the agents, we derive variants of Pop that can be connected with r-EF and that are Condorcet-consistent. However the most natural extension of Pop in such a context suffers from a computational burden. In practice, variants of Pop slightly improve Pop towards the selection goal. Nevertheless, the most interesting notion empirically is the outcome of the dynamics associated with r-EF swaps and FF-agreement moves which turns out to satisfy the goal of combining both selection and allocation.

Many interesting directions can be explored. Technically, some algorithmic questions remain open for our variants of Pop. Moreover, we have focused on pairwise comparisonsbased refinements of Pop but one could think about other types of refinements. To further understand how selection and allocation can be combined, an extensive study deserves to be initiated on the dynamics associated with r-EF swaps and FF moves. Concerning optimality, one could also consider minimizing the rank of the least happy agent and examine it under the lens of r-EF. Intuitively, r-EF seems to be very sensitive to strategic manipulation. This point deserves to be formally investigated. Finally, the extension to more than one project per agent or more than one agent per project is natural.

\section{Acknowledgements}

This work has been partially supported by the CNRS, via the project INS2I - 2021 - DeSIRe. 


\section{References}

[Abdulkadiroğlu and Sönmez, 1998] Atila Abdulkadiroğlu and Tayfun Sönmez. Random serial dictatorship and the core from random endowments in house allocation problems. Econometrica, 66(3):689-701, 1998.

[Abraham et al., 2004] David J. Abraham, Katarína Cechlárová, David F. Manlove, and Kurt Mehlhorn. Pareto optimality in house allocation problems. In Proceedings of the 15th International Symposium on Algorithms and Computation (ISAAC-04), pages 3-15, 2004.

[Abraham et al., 2007] David J. Abraham, Robert W. Irving, Telikepalli Kavitha, and Kurt Mehlhorn. Popular matchings. SIAM Journal on Computing, 37(4):1030-1045, 2007.

[Barberà et al., 2004] Salvador Barberà, Walter Bossert, and Prasanta K. Pattanaik. Ranking sets of objects. In Handbook of Utility Theory, pages 893-977. Springer, 2004.

[Benabbou et al., 2018] Nawal Benabbou, Mithun Chakraborty, Xuan-Vinh Ho, Jakub Sliwinski, and Yair Zick. Diversity constraints in public housing allocation. In Proceedings of the 17th International Conference on Autonomous Agents and MultiAgent Systems (AAMAS-18), pages 973-981, 2018.

[Beynier et al., 2019] Aurélie Beynier, Yann Chevaleyre, Laurent Gourvès, Ararat Harutyunyan, Julien Lesca, Nicolas Maudet, and Anaëlle Wilczynski. Local envy-freeness in house allocation problems. Autonomous Agents and Multi-Agent Systems, 33(5):591-627, 2019.

[Bouveret and Lang, 2008] Sylvain Bouveret and Jérôme Lang. Efficiency and envy-freeness in fair division of indivisible goods: Logical representation and complexity. Journal of Artificial Intelligence Research, 32:525-564, 2008.

[Bouveret et al., 2016] Sylvain Bouveret, Yann Chevaleyre, and Nicolas Maudet. Fair allocation of indivisible goods. In Handbook of Computational Social Choice, pages 284310. Cambridge University Press, 2016.

[Brams, 1990] Steven J. Brams. Constrained approval voting: A voting system to elect a governing board. Interfaces, 20(5):67-80, 1990.

[Bredereck et al., 2018] Robert Bredereck, Piotr Faliszewski, Ayumi Igarashi, Martin Lackner, and Piotr Skowron. Multiwinner elections with diversity constraints. In Proceedings of the 32nd AAAI Conference on Artificial Intelligence (AAAI-18), pages 933-940, 2018.

[Cechlárová and Fleiner, 2017] Katarína Cechlárová and Tamás Fleiner. Pareto optimal matchings with lower quotas. Mathematical Social Sciences, 88:3-10, 2017.

[Conitzer, 2009] Vincent Conitzer. Eliciting single-peaked preferences using comparison queries. Journal of Artificial Intelligence Research, 35:161-191, 2009.

[Cseh, 2017] Ágnes Cseh. Popular matchings. In Trends in Computational Social Choice, pages 105-122. AI Access, 2017.
[Elkind et al., 2017] Edith Elkind, Piotr Faliszewski, Piotr Skowron, and Arkadii Slinko. Properties of multiwinner voting rules. Social Choice and Welfare, 48(3):599-632, 2017.

[Faliszewski et al., 2017] Piotr Faliszewski, Piotr Skowron, Arkadii Slinko, and Nimrod Talmon. Multiwinner voting: A new challenge for social choice theory. In Trends in computational social choice, pages 27-47. AI Access, 2017.

[Foley, 1967] Duncan K. Foley. Resource allocation and the public sector. Yale Economic Essays, 7(1):45-98, 1967.

[Gan et al., 2019] Jiarui Gan, Warut Suksompong, and Alexandros A. Voudouris. Envy-freeness in house allocation problems. Mathematical Social Sciences, 101:104$106,2019$.

[Harless, 2018] Patrick Harless. Immediate acceptance and weak priorities: An adaptation that preserves efficiency and respects rank. Working paper, 2018.

[Hylland and Zeckhauser, 1979] Aanund Hylland and Richard Zeckhauser. The efficient allocation of individuals to positions. Journal of Political Economy, 87(2):293-314, 1979.

[Irving et al., 2006] Robert W. Irving, Telikepalli Kavitha, Kurt Mehlhorn, Dimitrios Michail, and Katarzyna E. Paluch. Rank-maximal matchings. ACM Transactions on Algorithms (TALG), 2(4):602-610, 2006.

[Irving, 2003] Robert W. Irving. Greedy matchings. Technical Report TR-2003-136, University of Glasgow, 2003.

[Kojima and Ünver, 2014] Fuhito Kojima and M Utku Ünver. The "Boston" school-choice mechanism: an axiomatic approach. Economic Theory, 55(3):515-544, 2014.

[McDermid and Irving, 2011] Eric McDermid and Robert W. Irving. Popular matchings: structure and algorithms. Journal of combinatorial optimization, 22(3):339-358, 2011.

[Ramezanian and Feizi, 2020] Rasoul Ramezanian and Mehdi Feizi. Stepwise ordinal efficiency for the random assignment problem. Journal of Mathematical Economics, 2020.

[Roth, 1985] Alvin E. Roth. The college admissions problem is not equivalent to the marriage problem. Journal of Economic Theory, 36(2):277-288, 1985.

[Shams et al., 2020] Parham Shams, Aurélie Beynier, Sylvain Bouveret, and Nicolas Maudet. Fair in the eyes of others. In Proceedings of the 24th European Conference on Artificial Intelligence (ECAI-20), pages 203-210, 2020.

[Shapley and Scarf, 1974] Lloyd Shapley and Herbert Scarf. On cores and indivisibility. Journal of Mathematical Economics, 1(1):23-37, 1974.

[Varian, 1974] Hal R. Varian. Equity, envy, and efficiency. Journal of Economic Theory, 9(1):63-91, 1974.

[Walsh, 2015] Toby Walsh. Generating single peaked votes. arXiv preprint arXiv:1503.02766, 2015. 